Nataša Ignjatović ${ }^{1}$

Faculté de Philosophie

Université de Niš
Оригинални научни рад

UDK 811.133.1'342:37.02

Примљено: 27.2.2020.

Ревидирана верзија: 7.4.2020.

Одобрено за штампу: 8.4.2020.

DOI: https://doi.org/10.46630/gsoc.24.2020.02

\title{
LES JEUX DE PRONONCIATION ET LA COHÉSION DU GROUPE-CLASSE DE FLE ${ }^{2}$
}

\begin{abstract}
Résumé : Le premier contact avec une nouvelle langue étrangère peut provoquer le sentiment d'insécurité, de mal à l'aise, de timidité, de manque de confiance, notamment chez les jeunes apprenants. Le système phonologique de la langue française ayant des dissemblances par rapport à celui de la langue serbe peut savérer difficile pour certains jeunes adolescents serbophones qui, découragés, ont tendance à se replier sur soi-même surtout si dans le groupe l'esprit de solidarité, tolérance et empathie ná pas été préalablement établi. Mais, si l’enseignant possède des compétences de gestion de groupe-classe, s'il rend son enseignement de la prononciation ludique et amusant, il va réussir à créer une bonne ambiance qui va contribuer à la cohésion du groupe-classe non seulement pendant les cours de français langue étrangère mais aussi une cohésion durable.

Pour montrer comment l'enseignant peut instaurer un esprit coopératif et développer la cohésion du groupe-classe à l'aide des activités de prononciation au début de l'apprentissage du français langue étrangère, dans cet article nous proposons quelques jeux possibles à utiliser en classe se référant aux éléments segmentaux et suprasegmentaux qui s’avèrent souvent difficiles pour les élèves serbophones.

Mots clés : prononciation, cohésion, adolescents, groupe-classe, FLE.
\end{abstract}

\section{Introduction}

Dans les établissements scolaires de l'enseignement primaire en Serbie, les élèves commencent à apprendre une première langue étrangère en première classe, l'anglais par défaut et presque sans exception, tandis qu'en cinquième classe ils peuvent opter pour une deuxième langue étrangère, c'est à dire l'allemand, le français, le russe ou l'italien. Depuis la rentrée scolaire 2007, les élèves et leurs parents font choix entre deux langues proposées. Étant donné qu'un grand nombre délèves choisit l'allemand, les élèves de toutes les classes qui choisissent le français étant moins nombreux, se retrouvent dans un groupe-classe composé d'une vingtaine délèves de toutes les classes

\footnotetext{
${ }^{1}$ natasa.ignjatovic@filfak.ni.ac.rs

${ }^{2}$ Cet article est rédigé dans le cadre du projet scientifique Les langues, les littératures et les cultures romanes et slaves en contact et en divergence № 81/1-17-8-01 financé par la Faculté de Philosophie de l'Université de Niš, l'Agence universitaire de la Francophonie (AUF) et l'Ambassade de France en Serbie.
} 
de la cinquième ${ }^{3}$. Cela fait que ces élèves se retrouvent dans un nouveau contexte, avec de nouveaux camarades, face à une nouvelle langue étrangère. Sachant quà cet âge-là les adolescents passent par une période de développement fragile, la puberté, la rencontre avec de nouveaux camarades et avec une nouvelle langue étrangère les met dans une position défavorable, surtout les plus timides. Pour pouvoir créer une ambiance amicale, aider les élèves à établir une relation de confiance mutuelle, l'enseignant pourrait recourir aux activités ludiques d'enseignement, surtout les activités et les jeux de prononciation afin de créer un groupe-classe soudé.

\section{La cohésion du groupe}

La cohésion sociale ou la cohésion du groupe veut dire que dans une communauté, un groupe il y a un lien important entre ses membres (Žegarac et al. 2016 : 26). On trouve l'explication dans le dictionnaire Larousse : «Propriété d'un ensemble dont toutes les parties sont solidaires ; solidarité [...]» (Larousse). Ceci dit que le bon fonctionnement des groupes sociaux est conditionné par les valeurs universelles, telles que le respect, la solidarité, l'empathie et la tolérance. Dans ce contexte, la rencontre avec une nouvelle langue étrangère, ici le français, c'est la rencontre avec l'altérité. Les travaux du Conseil de l'Europe destinés à promouvoir les valeurs des citoyens démocratiques, des droits de l'homme, les principes de l'égalité et de la cohésion sociale, représentent l'apprentissage des langues étrangères en tant qu'un facteur véhiculaire et médiateur dans l'appropriation de ces valeurs. Le Conseil de l'Europe considère l'apprentissage des langues étrangères comme atout principal de la formation de futurs citoyens démocratiques :

«C'est un processus participatif développé dans des contextes différents, mais qui, entre autres :

- prépare les hommes et les femmes à jouer un rôle actif dans la vie publique et à forger leur propre destinée ainsi que celle de leur société ;

- vise à inculquer une culture de droits de l'homme ;

- prépare les gens à vivre dans une société pluriculturelle et à reconnaître le droit à la différence avec intelligence, sensibilité, tolérance et moralité ;

- renforce la cohésion sociale, la compréhension mutuelle et la solidarité » (Conseil de l'Europe, 1999 cité dans Starkey 2003 : 69-70).

C’est-à-dire que l'apprentissage des langues étrangères permet le rapprochement de l'individu vers l'altérité, le développement des valeurs, telles que la solidarité, la tolérance, lempathie qui assurent ensuite le bon fonctionnement du groupe, la stabilité et la cohésion. En apprenant une langue étrangère, pourtant, dans cette rencontre avec l'altérité, lélève peut ressentir du rejet provoqué par les différences phonétiques entre les deux langues. Car, la prononciation est personnelle, la capacité d'imiter la prononciation d'une nouvelle langue dépend des facteurs biologiques, psychologiques et cognitifs (Lauret 2007). De plus, Lauret constate que travailler

\footnotetext{
${ }^{3}$ Le nombre délèves dans ces groupes-classes varient entre quinze et vingt-cinq.
} 
sur la prononciation comprend la prise en compte de facteurs variés, tels que « la personne, la souplesse de son ego [...], sa motivation, son attention auditive, ses capacités vocales, son plaisir du jeu, [...]» (Lauret $2007: 16$ ).

Le rôle de l'enseignant serait de choisir les activités afin de rapprocher la prononciation du français, d'inciter d'abord l'acceptation du nouveau, de gérer les comportements des élèves dans le groupe, de construire la cohésion dans la classe. Les apprenants aidés par l'enseignant devraient pouvoir accepter et s'approprier cette nouvelle identité en classe de langue, cet autre " moi » qui parle une langue étrangère, ici, le français, celui qui prononce les nouveaux sons, cette nouvelle musique d'une autre langue. Lauret affirme que la prononciation est aussi une question de l'identité :

« Adopter la différence phonétique d'une langue étrangère ne pose généralement pas de problèmes aux enfants. Par contre chez les adolescents et les adultes, la différence fait souvent peur, comme si elle risquait de fragiliser la personne. On peut se sentir ridicule à articuler des sons qui ne nous sont pas habituels. Ils sont donc importants de comprendre que l'adoption d'une nouvelle musique et de nouveaux sons ne représente aucun risque identitaire ; savoir être différent est au contraire un jeu, un plaisir et un enrichissement » (Lauret 2007: 21).

C'est à l'aide de l'enseignant, en jouant avec les nouveaux sons et la musique d'une nouvelle langue que l'apprenant pourrait adopter une nouvelle identité, réservée pour les cours de français et qu'il va partager avec ses camarades en classe de français.

Une recherche faite auprès des étudiants de langue et littérature française sur les représentations et attitudes envers la France et la langue française démontre l'importance de la sonorité d'une langue car la plupart des étudiants interrogés $(64,3 \%)$ ont dit qu'ils trouvaient la langue française "belle » et " mélodieuse » (Ignjatović, Simović 2015: 415). La sonorité d'une langue étrangère est alors reconnue comme caractéristique primaire, c'est un trait perçu immédiatement qui peut s'avérer motivant (selon le groupe interrogé) mais aussi démotivant, dû aux nombreux facteurs. Quand la sonorité d'une nouvelle langue plaît aux élèves cela peut les inciter à vouloir acquérir la bonne prononciation du français.

\section{Les systèmes phonologiques et prosodiques de deux langues : le français et le serbe}

Le système phonologique de la langue française est caractérisé par la tension, la labialité, l'antériorité et la nasalité tandis que la prononciation des sons de la langue serbe est plus relâchée, les sons sont plutôt postérieurs et moins arrondis, les voyelles sont caractérisées par la mélodie (Šotra 2006 : 73). En français standard il y a 16 voyelles (12 voyelles orales et 4 voyelles nasales), 3 semi-voyelles et 17 consonnes, quoique le nombre des voyelles puisse varier selon auteurs, selon leur choix de représenter le système phonologique du français standard ou bien en se référant à l'usage. Par exemple, on trouve dans le classement des Léon 16 voyelles : très fermées $[i, u, y]$, fermées [e, ø, o, õ], moyennes [ə], ouvertes $[\varepsilon, œ, \diamond, \tilde{\varepsilon}, \tilde{a}]$ et très ouvertes 
[a, a, ã] (Léon M. et Léon P. 2009: 22), tandis que chez Abry et Veldeman-Abry, on trouve 13 voyelles : hautes fermées [i, u, y], mi-hautes mi-fermées [e, ø, o], mi-basses

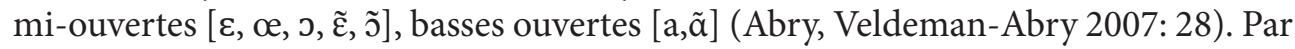
contre, la langue serbe possède cinq voyelles : /a/, /e/, /i/, /o/, /u/ et 25 consonnes. Les élèves serbophones ont des difficultés à prononcer certaines voyelles françaises ce qui peut être dû aux dissemblances liées aux systèmes vocaliques de deux langues. Quant au système consonantique du français, il ne représente pas de difficultés de prononciation aux locuteurs serbophones, à part la consonne [R] (Točanac 1997 : 34).

En ce qui concerne la prosodie de deux langues, la principale différence repose dans laccentuation et le rythme. Dans la langue serbe chaque mot est porteur de l'accent tandis qu'en français le mot porte l'accent uniquement s'il est pris isolément, s'il s'agit d'un groupe de mots ou groupe rythmique, la dernière syllabe du groupe est porteur de l'accent. D’après Léon « L'accentuation est le résultat d’un effort expiratoire et articulatoire qui se manifeste par une augmentation physique de longueur, d'intensité et éventuellement un changement de fréquence en passant de syllabe inaccentuée à accentuée ou au cours de lévolution de la syllabe accentuée " (Léon 2011: 154). Donc, si le mot est pris isolément, l'accent est mis sur la dernière syllabe tandis que les syllabes précédentes du mot sont inaccentuées. Laccent est donc oxytonique, dans le français standard (Léon 2011 : 155). Contrairement à la langue française, l'accent de la langue serbe est polytonique. Ainsi, les voyelles possèdent quatre accents : l'accent court descendant, l'accent court montant, l'accent long descendant, l'accent long montant, dont le ton varie à l'intérieur d'une syllabe. Ces différences provoquent les erreurs qui se produisent dans la prononciation des apprenants serbophones du français langue étrangère car en français c'est la dernière syllabe du groupe rythmique qui porte accent tandis qu'en serbe il s'agit d'une unité accentuelle. Šotra constate que « la mélodie de la phrase française dépend de l'accent final du groupe rythmique alors que l'unité accentuelle détermine la mélodie de la phrase serbe [...] (Šotra 2006 : 119). Selon Lauret, il serait essentiel que les apprenants étrangers s'approprient le rythme, l'accentuation et l'allongement de la dernière syllabe car cela contribue à l'intelligibilité (Lauret 2007 : 52).

Après avoir comparé les systèmes phonologiques et prosodiques de deux langues, on peut constater qu'il serait important de favoriser l'acquisition de la mélodie du français, c'est-à-dire le rythme, l'accentuation et l'intonation dès les premières heures de l'apprentissage en rajoutant ensuite le travail sur les segments notamment sur ceux qui posent des difficultés : la voyelle fermée [y], les voyelles à

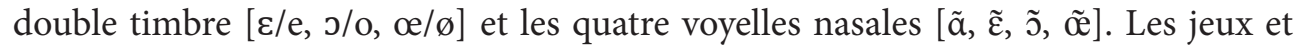
les activités ludiques de prononciation permettent à l'enseignant d’éveiller le plaisir chez les jeunes apprenants. Comme le constate Michel Billières sur son blog « la phonétique rime avec ludique » (Billières 2017). 


\section{Les jeux et les activités ludiques}

Les jeux en général, contribuent au développement intellectuel des enfants. Les sollicitations que les jeux imposent permettent aux enfants d'utiliser les gestes, le corps, les émotions et les capacités cognitives. L'enfant entre en contact avec les autres, joue des rôles différents, doit communiquer avec les autres et ainsi il développe ses capacités affectives et cognitives (Silva 2008: 24-25). Ce type d'activité qui demande aux élèves de coopérer, joue un rôle important dans le processus de socialisation des enfants et des adolescents. L'une des stratégies socio-affectives, la stratégie de coopération qui comprend l'interaction avec les autres membres du groupe afin de réaliser une tâche commune (Cyr 1998: 56) est essentielle lors des activités ludiques. La prise de parole est alors facilitée car les élèves négocient d'abord en groupe ensemble avant de donner la réponse. Ainsi, les élèves développent d'abord la confiance en soi et ensuite, en coopérant avec leurs camarades ils développent également lesprit solidarité, lempathie, le respect ce qui renforce le lien entre eux et établit la cohésion du groupe-classe.

Quant au choix des activités ludiques à intégrer en classe, Weiss propose des pistes de réflexion aux enseignants qui devraient les guider. En fait, il faut prendre en compte le niveau des élèves, lobjectif de cette activité, le prérequis, la modalité (en groupe ou individuellement), le type d'activité (production guidé/ouverte/libre), dans quelle étape du cours il faut faire cette activité, comment la préparer, combien de temps prévoir, etc. (Weiss 2002: 10).

Nous allons proposer quelques activités de prononciation à intégrer en classe, en recourant aux jeux proposés dans la littérature en les modifiant selon les besoins des élèves serbophones et en rajoutant des exploitations possibles en classe de FLE.

\subsection{Quelques jeux de prononciation à intégrer en classe}

Les activités ludiques et les jeux de prononciation en classe ont un double intérêt : d'abord, le jeu est inhérent à l'enfant, donc, c'est en jouant qu'il apprend mieux; ensuite, le jeu l'aide à dépasser ses angoisses provoquées par la prononciation d'une langue différente que la sienne pour enfin mieux l'accepter. De plus, comme on a pu le constater plus haut, c'est en coopérant que peut s'instaurer la cohésion du groupeclasse. Dans cette section, nous allons donc présenter les jeux de prononciation pour un groupe classe du niveau débutant, quon peut utiliser à la fois comme les activités de brise-classe en classe de cinquième et à la fois comme les activités de prononciation et l'appropriation des sons et de la prosodie. Nous allons donner quelques exemples de jeux qu'on pourrait utiliser en classe.

1) Un jeu de prononciation proposé par Haydée Silva : le jeu de bingo, pour travailler les paires doppositions phonétiques $[\mathrm{y}] / \mathrm{u}]$. Elle propose un corpus des paires de mots d'oppositions phonétiques [y] et [u] à utiliser pour faire le jeu de bingo. C'est-à-dire qu'il faut constituer deux groupes délèves et leur distribuer les mots ayant ces voyelles. L'enseignant lit les mots selon son choix de mots préalablement mis en désordre et ensuite les élèves cochent ces mots sur la liste (Silva 2008: 52). 
2) Le jeu que nous proposons vise l'acquisition des voyelles fermées [i, u, y] mais en variant les voyelles et en inventant les phrases, ce jeu peut être destiné à l'acquisition des autres voyelles aussi. De plus, en variant l'intonation (montante/ descendante) on intègre aussi bien le travail sur l'intonation. Les élèves sont debout, en cercle, l'enseignant prononce les phrases en insistant sur les voyelles à travailler. Les élèves prononcent une phrase à leur tour de rôle. Ensuite, comme ce sont des mini-dialogues, les élèves peuvent faire un jeu de rôle. Il s'agit des sons à acquérir qui sont mis en contexte, ce qui est très important. Voici quelques exemples : L'as-tu vu? Il est où ? Il est sous la table? Il est sur la chaise? Que dis-tu? Tu as mal au genou? Tu vois un hibou? Es-tu sûr?

Ces phrases servent à titre d'exemple. Il faut donc trouver de courtes phrases, ayant les sons en question et puis proposer quelques variantes :

Variantes 1 : Les élèves prononcent en rallongeant la voyelle finale de la syllabe ouverte: Que dis-tuuuuu? Il est oùoùoù ? ou bien en rallongeant la voyelle de la syllabe fermée : Tu es sîûûिûr ? etc. De cette manière la, en jouant, les élèves articulent les voyelles et mettent en valeur la syllabe finale accentuée.

Variante 2 : jeu de miroir : les élèves prononcent ces phrases l'une après l'autre en même temps en se regardant et en essayant de rester focalisés. Ou bien, un élève peut prononcer une phrase en regardant l'autre et ensuite l'autre élève répète la phrase entendue. C’est le moment de détente, de rire, de l'ambiance positive qui se crée tandis que les élèves, une fois retrouvés dans la même situation, coopèrent, se focalisent à réaliser leurs tâches et la timidité disparait progressivement.

3) Voici encore un jeu, très connu par toutes les générations, qui marche très bien en groupe-classe, c'est un jeu de coopération qui permet détablir la cohésion du groupe-classe. De plus, ce jeu est facile à intégrer après avoir fixé l'objectif phonétique. Voici un exemple d'exploitation : si l'on veut travailler sur la perception des voyelles nasales, par exemple, on peut faire écouter une comptine ou une chanson qui contient les voyelles nasales, en veillant à ce que le débit soit lent pour que les élèves puissent repérer avec une certaine facilité les sons en question. On donne la consigne avant l'écoute : si la musique s'arrête au moment où vous entendez une voyelle nasale, il faut s'assoir, si vous entendez un autre son au moment où la musique s'arrête, il faut continuer à circuler autour des chaises. Les adolescents aiment bien les jeux qui démarrent leur esprit de compétition, alors ce jeu, étant facilement exploitable peut être utilisé pour travailler aussi bien sur la perception et l'identification des voyelles nasales que sur d'autres segments qui s’avèrent difficiles pour les élèves. De plus, ce jeu fait bouger les élèves, ils ne restent pas assis. Étant donné que les groupes délèves ne sont pas nombreux, de quinze à vingt élèves, le jeu peut être facilement géré par l'enseignant, sauf s'il ne s'agit pas d'une petite salle ou bien si le nombre dépasse vingt élèves par groupe.

4) On peut proposer aussi Le jeu de cartes ayant comme objectif le travail sur les syllabes, le rythme et l'accentuation. Vu les principales différences existant entre les systèmes prosodiques de deux langues, il serait nécessaire de travailler sur le développement de la compétence prosodique dès le début de l'apprentissage du 
français. Pour montrer que la dernière syllabe du mot isolé ou la dernière syllabe du groupe porte l'accent, nous proposons le jeu qui pourrait être réalisé à la suite des activités de perception auditive et de repérage.

L'enseignant propose trois séries de cartes : la première avec un seul mot, la deuxième avec des syntagmes et la troisième série avec des phrases. Par exemple, sur une carte de la première série on peut lire le mot "la voiture " où les trois lettres qui correspondent aux sons prononcés et qui font la dernière syllabe du mot seraient représentées en couleur rouge pour marquer la place de l'accent. La carte de la deuxième série aurait le syntagme " la voiture rouge " ou les lettres prononcées $\mathrm{du}$ mot " rouge " seraient mis en couleur pour marque la place de laccent. Finalement, la première carte de la troisième série aurait la phrase « La voiture rouge bouge beaucoup » où l'on marque en couleur les lettres qui correspondent aux sons prononcés, porteur de l'accent, du groupe nominal " la voiture rouge » et ainsi que du groupe verbal «bouge beaucoup» On choisit la rime exprès afin de faciliter la mémorisation, car c'est en se rappelant des exemples que les élèves peuvent mieux appliquer les mêmes "règles » en prononçant ou en lisant d'autres exemples. Les élèves, étant divisés en groupes, reçoivent les mêmes cartes par groupe, cinq cartes de chaque série. L'enseignant choisit un mot de la première série, montre la carte avec ce mot aux groupes délèves et leur donne comme consigne de trouver la carte correspondante de la deuxième et troisème série. La première équipe qui réussit à trouver les bonnes cartes gagne le jeu. Ensuite, l'enseignant approfondit le travail en demandant aux élèves d'observer les cartes, de lire les mots en rallongeant les syllabes mis en couleur rouge.

5) Le jeu qui relie les phonèmes avec les graphèmes correspondants peut être proposé aux élèves après les activités de perception, repérage et discrimination des mots ayant le son étudié. Si le son étudié est par exemple /o/, on demande aux élèves de trouver dans la boîte à mots toutes les cartes de mots ayant ce son. Les élèves se mettent en groupe, on leur propose des boîtes avec les cartes, les cartes étant les mêmes dans chaque boîte et on leur accorde le temps pour travailler. On fait une mise en commun et les membres du groupe collent les mots au tableau. Après l'étude des mots, l'enseignant aide les élèves à repérer les graphies. Voici quelques exemples de mots que les élèves doivent trouver dans la boîte : dodo, soleil, orange (les mots faciles à repérer par analogie avec la même lettre en serbe); bateau, cadeau, Australie (les mots plus difficiles à repérer à cause de leurs graphèmes). Après la mise en commun, l'enseignant peut entourer les graphèmes avec un crayon de couleur. Le gagnant est le groupe qui trouve tous les mots ayant le son /o/ se retrouvant dans la boîte. On peut appliquer la même activité pour faire fixer les graphèmes de tous les phonèmes étudiés. Ce jeu est amusant est facilement exploitable en classe.

Nous avons proposé ces activités connaissant les difficultés typiques de prononciation des élèves serbophones. L'enseignant va sûrement cibler son travail en fonction des résultats du diagnostic obtenus sur la prononciation de ses élèves. 


\section{Conclusion}

La prononciation d'une langue étrangère peut représenter le plaisir ou l'obstacle pour les jeunes apprenants au début de l'apprentissage, notamment si les élèves se retrouvent dans un nouveau groupe-classe. Nous avons montré quelques jeux de prononciation que l'enseignant pourrait mettre en place car le jeu permet aux élèves de se détendre, incite la motivation, éveille le plaisir. Dans une telle ambiance les élèves sont prêts à apprécier la nouvelle mélodie, à mieux accepter les nouveaux sons et à dépassser l'angoisse en jouant avec leurs camarades. Les exemples de jeux que nous avons choisis visent l'acquisition des sons de la langue française qui n'existent pas dans le système phonologique de la langue serbe ainsi que les caractéristiques prosodiques qui s'avèrent difficiles à surmonter par des élèves. De plus, les jeux que nous avons montrés peuvent aussi être réadaptés et utilisés pour enseigner pratiquement tous les éléments segmentaux et suprasegmentaux du français. Nous considérons que les élèves peuvent mieux acquérir la bonne prononciation en jouant, cela leur permet aussi de coopérer tout en se focalisant sur la réalisation de la tâche commune, ce qui contribue en même temps à la création d'un groupe-classe soudé.

\section{Bibliographie}

Abry, D., Veldeman-Abry J. (2007) La phonétique: audition, prononciation, correction. Paris: CLÉ International.

Billières, M. (2017) «Phonétique rime avec ludique».https://www.verbotonale-phonetique. com/phonetique-rime-ludique/

Cyr, P. (1998) Les stratégies d’apprentissage. Paris : CLÉ International.

Dictionnaire Français en ligne - Larousse : https:/larousse.fr/dictionnaires/francais/

Gudurić, S. (2009) Osnovi fonetike s fonologijom francuskog jezika. Beograd: Zavod za udžbenike i nastavna sredstva.

Guimbretière, É. (1996) Phonétique et enseignement de l'oral. Paris : Didier/Hatier.

Ignjatović, N. (2018) Phonétique et orthographe 1 pour les étudiants de FLE, cahier d'exercices. Niš: Filozofski fakultet.

Ignjatović, N., Simović V. (2015) «Les images stéréotypées chez les étudiants de langue et littérature françaises ", Les Études françaises aujourd'hui : tradition et modernité : 409-424.

Léon, M., Léon, P. (2009) La prononciation du français. Paris: Armand Colin.

Léon, P. (2011) Phonétisme et prononciations du français. Paris: Armand Colin.

Lauret, B. (2007) Enseigner la prononciation du français : questions et outils. Paris: Hachette.

Neuner, G., Parmenter L., Starkey, H., Zarate, G. (2003) La compétence interculturelle. Strasbourg : Conseil de l'Europe.

Silva, H. (2008) Le jeu en classe de langue. Paris: CLÉ International. 
Simović, V. (2012) «Igra u učenju/nastavi stranog jezika », Filologija i Univerzitet : 678687.

Subotić, Lj., Sredojević D., Bjelaković I. (2012) Fonetika i fonologija: ortografska i ortoepska norma standardnog srpskog jezika. Novi Sad: Filozofski fakultet.

Točanac-Milivojev, D. (1997) Metode u nastavi i učenju stranog jezika. Beograd: Zavod za udžbenike i nastavna sredstva.

Šotra, T. (2006) Kako progovoriti na stranom jeziku. Beograd: Zavod za udžbenike i nastavna sredstva.

Šotra, T. (2010) Didaktika francuskog kao stranog jezika. Beograd: Filološki fakultet.

Žegarac, N., Kišjuhas, A., Koprivica I. (2016) Pojmovnik kulturno kompetentne prakse. Novi Sad: Pokrajinski zavod za socijalnu zaštitu.

Weiss, F. (2002) Jouer, communiquer, apprendre. Paris: Hachette.

\section{ИГРЕ ИЗГОВОРА И КОХЕЗИЈА ГРУПЕ НА ЧАСОВИМА ФРАНЦУСКОГ КАО СТРАНОГ ЈЕЗИКА}

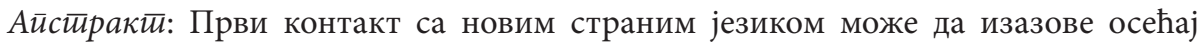
несигурности, нелагоде, стидљивости, неповерења, посебно код млађих ученика. Услед разлике између два фонолошка система, француског и српског, изговор француског језика може бити тежак за неке ученике чији је матерњи језик српски, те обесхрабрени, имају обичај да се повуку у себе, посебно уколико унутар саме групе није успостављен однос толеранције, солидарности и емпатије. Међутим, ако наставник поседује потребне вештине управљања разредом, уколико учење изговора учини забавним, претпостављамо да ће успети да створи повољну атмосферу која ће допринети не само кохезији групе на часовима француског језика него и трајној кохезији унутар те групе. Како бисмо показали на који начин наставник може да успостави сарадњу и развије кохезију у групи, уз помоћ активности изговора на почетку учења француског језика, предложићемо неке од могућих игара које се односе на сегментне и супрасегментне елементе који често представљају потешкоће србофоним ученицима.

Кључне речи: изговор, кохезија, адолесценти, разред, француски као страни језик. 